
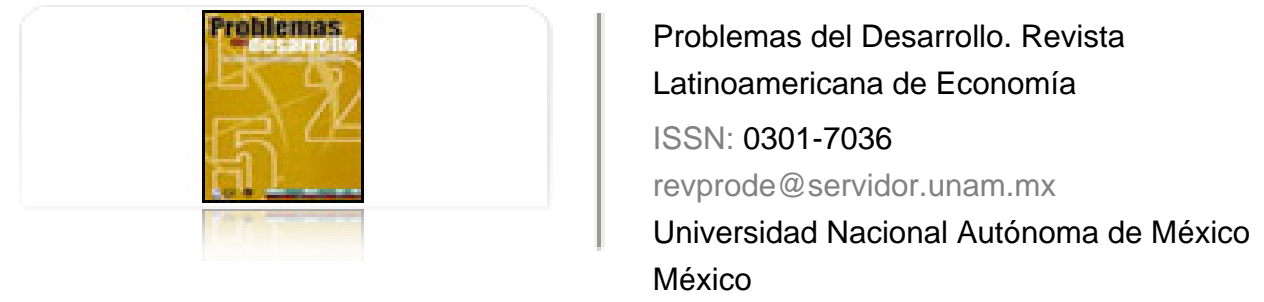

Valdivia López, Marcos; Delgadillo Macías, Javier; Galindo Pérez, Carlos Nuevos patrones espaciales en las derramas de empleo en la zona metropolitana de la Ciudad de México

Problemas del Desarrollo. Revista Latinoamericana de Economía, vol. 41, núm. 163, octubrediciembre, 2010, pp. 99-117

Universidad Nacional Autónoma de México

Distrito Federal, México

Disponible en: http://www.redalyc.org/articulo.oa?id=11819762006

Cómo citar el artículo

- Número completo

- Más información del artículo

Página de la revista en redalyc.org

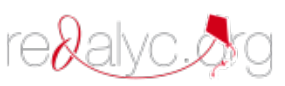

Sistema de Información Científica

Red de Revistas Científicas de América Latina, el Caribe, España y Portugal Proyecto académico sin fines de lucro, desarrollado bajo la iniciativa de acceso abierto 
Revista Problemas del Desarrollo, 163 (41), octubre-diciembre 2010

\title{
Nuevos PATRONES ESPACIALES EN LAS DERRAMAS DE EMPLEO EN LA ZONA METROPOLITANA DE la CiUdad de MÉXico
}

\author{
Marcos Valdivia López*, Javier Delgadillo Macías** y Carlos Galindo Pérez*** \\ Fecha de recepción: 16 de agosto de 2010. Fecha de aceptación: 15 de octubre de 2010.
}

\section{RESUMEN}

En la Zona Metropolitana de la Ciudad de México (ZMCM) la generación de derramas en el empleo puede estar asociada, entre otros factores, a la adopción de nuevas tecnologías (vía inversión extranjera directa), a la inmigración de gente calificada o talentosa, y/o a la presencia de centros de investigación y desarrollo. En este trabajo se analiza el papel de las derramas en el empleo del sector manufacturero y en el sector de servicios profesionales a través de dos grupos que hemos clasificado como creativos y tecnológicos. El resultado central de este trabajo es que los patrones de derrama de empleo se han modificado durante las últimas dos décadas y han estado condicionados por la intensificación del proceso de terciarización que ha experimentado la ZMCM. En particular se propone que este proceso de reconversión económica de la metrópoli ha generado que los efectos derrama de empleo operen en distancias cortas de difusión espacial.

\section{PALABRAS CLAVE}

Derramas, difusión espacial, empleo profesional, autocorrelación espacial.

* Investigador en el Centro Regional de Investigaciones Multidisciplinarias de la UNAM. Correo electrónico: marcosv@ correo.crim.unam.mx

** Investigador en el Instituto de Investigaciones Económicas de la UNAM. Correo electrónico: javierdelma@gmail.com

*** Técnico del Centro Regional de Investigaciones Multidisciplinarias de la UNAM. Correo electrónico: carlosgp@correo. crim.unam.mx 
Marcos Valdivia L., Javier Delgadillo M. y Carlos Galindo P.

\section{Summary}

In the Mexico City Metropolitan Zone (MCMZ) the generation of employment spillovers may be associated with the adoption of new technologies (via foreign direct investment), the immigration of qualified or talented people, and/or the presence of research and development centers, among other factors. In the present paper we analyze the role of employment spillovers in the manufacturing and professional service sectors using two groups which we have classified as creative and technological. The core result we reach is that the patterns of employment spillovers have changed over the last two decades and have been conditioned by the intensification of the tertiarization process experienced by the MCMZ. In particular, it is proposed that this process of economic conversion in the metropolis has meant that the spillover employment effects occur over short distances of spatial diffusion.

Key words: spillovers, spatial diffusion, professional employment, spatial self-correlation.

\section{Résumé}

Dans la zone métropolitaine de la ville de Mexico (ZMVM), la génération de gisements d'emploi peut être associée, entre autres facteurs, à l'adoption de nouvelles technologies (au travers d'investissement étranger direct), à l'immigration de gens qualifiés ou talentueux, et/ou à la présence de centres de recherche et de développement. Dans ce travail est analysé le rôle des gisements dans l'emploi du secteur manufacturier et dans le secteur des services professionnels à travers de deux groupes que nous avons classés comme créatifs et technologiques. Le résultat central de ce travail est que les schémas de gisement d'emploi se sont modifiés au cours des deux dernières décennies et ont été conditionnés par l'intensification du processus de tertiarisation qu'a connu la ZMVM. Il est notamment avancé que ce processus de reconversion économique de la métropole a provoqué que les effets gisement d'emploi opèrent sur de courtes distances de diffusion spatiale.

Mots clés : gisements, diffusion spatiale, emploi professionnel, auto-corrélation spatiale.

\section{Resumo}

Na Zona Metropolitana da Cidade do México (ZMCM) a geraçáo de efeito-derramamento sobre o emprego pode estar associada, entre outras coisas, a adoção de novas tecnologias (via investimento estrangeiro direto), a imigraçáo de pessoas qualificadas e talentosas, e / ou a presença de centros de pesquisa e desenvolvimento. Este artigo analisa o papel do efeito-derramamento do emprego no setor manufatureiro e no setor de serviços profissionais através de dois grupos que qualificamos como criativos e tecnológicos. $O$ resultado central deste trabalho é que os padróes do efeito-derramamento do emprego se modificaram ao longo das últimas duas décadas e têm sido condicionados pela intensificaçáo do processo de terceirizaçáo que se experimentou na ZMCM. Em particular, se propóe que este processo de reconversão econômica da metrópole que gerou repercussóes no sentido que o efeito-derramamento do emprego opere em distâncias curtas de difusão espacial.

Palavras-chave: efeito-derramamento, difusão espacial, emprego profissional, autocorrelação espacial.

\section{墨西哥城都市市区就业的新空间格局}

在, 墨西哥城都市市区 (ZMCM) 就业的外溢可关联于新技术的采用（通过外商直接 投资），高技术和人才的移民，和研究与开发中心的存在。本文通过两个叫做创 作群体和技术群体小组的观察, 分析了在制造业和专业服务行业中的就业溢出效 应。中心结论是, 在过去二十年内, 在大都市区第三产业加强的影响下, 就业模 式具有明显的改变。我们认为大度市区的经济转型使就业溢出在短距离的空间扩 散.

关键词：溢出效应, 空间扩散, 专业就业, 空间关联。 


\section{INTRODUCCIÓN}

En este trabajo se analizan las derramas en empleo que las aglomeraciones económicas de la Zona Metropolitana de la Ciudad de México (ZMCM) han producido en el sector manufacturero y de servicios profesionales durante los últimos años. Para identificar y evaluar las derramas en empleo, se recurre al uso de técnicas de estadística espacial y a estimaciones econométricas espaciales, en las que se utilizan como proxies de derramas tanto: $i$ ) al "cluster horizontal" correspondiente al sector económico analizado en una delegación o municipio de la ZMCM, y ii) el crecimiento del empleo de un conjunto de microrregiones que pueden considerarse vecinas respecto a cada una de las delegaciones y municipios de la ZMCM. Asimismo, se presenta un ejercicio de segmentación del sector de servicios profesionales para identificar a una clase creativa y una clase técnica y observar que su localización territorial presenta una fuerte dependencia espacial en la ZMCM. Los indicadores utilizados en esta investigación corresponden al personal ocupado a nivel de sector, rama y subrama reportados por los censos económicos 1993, 1999 y 2003 del INEGI.

El principal hallazgo de esta investigación fue encontrar que el proceso de terciarización experimentado por la ZMCM durante las últimas dos décadas, ha provocado que las derramas de empleo (en el sector servicios) operen sobre distancias cortas de difusión espacial, proceso que contrasta con lo ocurrido en la etapa previa, cuando la concentración de ramas manufactureras de la ZMCM estaba asociada a derramas de empleo que operaban bajo distancias "largas". Es decir, la terciarización en la ZMCM ha provocado que cuando se genera una derrama de empleo por concentración económica, la nueva unidad de empleo que aparece a consecuencia de derramas por actividad terciaria se localiza ahora mucho más cercana en distancia que la generada anteriormente cuando predominaba la concentración económica por actividad industrial. Este resultado puede tener importantes implicaciones en materia de diseño de política de empleo regional.

\section{NOCIÓN DE DERRAMAS}

La noción de derrama a la que se alude en este trabajo está estrechamente relacionada con la discusión sobre economías externas en la teoría económica. El concepto de economías externas fue originalmente planteado por Marshall (1920), quien las definió como las ventajas que un productor puede obtener al localizarse cerca de otros productores de la misma industria.

Tres argumentos se han señalado para explicar la presencia de economías externas: a) la concentración geográfica ofrece proveedores de insumos en tiempos y distancias más cortas; $b$ ) la presencia de mercados laborales condensados que proveen mano de obra especializada; $\mathrm{y}$ c) la proximidad física que facilita la difusión e intercambio de tecnología e información. La noción de derramas utilizada en este trabajo, tiene que ver precisamente con este último elemento: el de la difusión. 
Marcos Valdivia L., Javier Delgadillo M. y Carlos Galindo P.

Debe señalarse que las economías externas han sido difíciles de formalizar dentro de un modelo económico convencional. De hecho, no fue sino hasta hace algunos años y con la aparición de la Nueva Geografía Económica (NGE), que algunos de los elementos que impulsan la formación de economías externas pudieron ser formalizados (Fujita-KrugmanVenables, 1999). Sin embargo, debe también señalarse que los modelos de la NGE tampoco han incorporado de manera sistemática las derramas tecnológicas e informativas, como sus propios precursores han reconocido (Krugman, 1991). Esto ha sido un elemento importante que ha prevenido, hasta hace poco, considerar o medir las derramas cuando se analizan las economías externas. Incluso ésta pareciera ser la tónica, entre los diversos estudios empíricos sobre aglomeraciones económicas y sus efectos espaciales que pueden encontrarse para el caso mexicano (Díaz, 2003; Ruiz, 2005; Garza, 2006; Assuad y Quintana, 2008), en los cuales también se ha otorgado poca atención a los efectos de derrame o spillover ${ }^{1}$ que están asociados al concepto de cluster económico.

Con el auge que en años recientes ha tenido la estadística espacial y, en particular la econometría espacial, en los estudios regionales, algunos autores no han dudado en señalar que las derramas que pueden estar presentes en diversos procesos económicos (por ejemplo, productividad, empleo, innovación, etcétera) son factibles hoy en día de medirse (Fingleton, 2003). A pesar de que este entusiasmo puede encontrar diversas críticas en la literatura (predominantemente en la NGE), adoptamos esta estrategia metodológica en el presente trabajo para analizar el papel de la derramas en la generación de empleo en la ZMCM y cómo éstas han cambiado durante las últimas dos décadas.

Este trabajo sitúa como unidad de análisis espacial al conjunto de unidades político-administrativas que integran la ZMCM (Mapa 1). Enfocarse al análisis de la ZMCM se fundamenta por: $i$ ) ser el espacio de mayor jerarquía urbana en el país; ii) ser una de las metrópolis mexicanas que aún mantiene su dinamismo económico por el peso de sus actividades productivas; iii) por su crecimiento sociodemográfico que le ha llevado a integrar una amplia zona metropolitana: 16 delegaciones del Distrito Federal, $59 \mathrm{mu}$ nicipios del Estado de México y uno de Hidalgo (CONAPO, 2007 y Gaceta Oficial del Distrito Federal, 23 de enero, 2005).

\section{METODOLOGÍA DE MEDICIÓN DE LAS DERRAMAS DE EMPLEO}

En cuanto al procedimiento metodológico, se recurre al uso de una serie de técnicas desarrolladas desde la econometría espacial para estudiar las derramas (Anselin, 2003; Fingleton, 2003). En particular, destaca el uso de una matriz de pesos entre unidades regionales $(W)$ para estudiar efectos espaciales, que pueden estar asociados a las derramas en general.

El término spillover tiene varias acepciones: derramas, efecto lateral, efectos externos, externalidades, entre otros. Aquí se asume como sinónimo de derramas (cfr: CEPAL, 2004). 


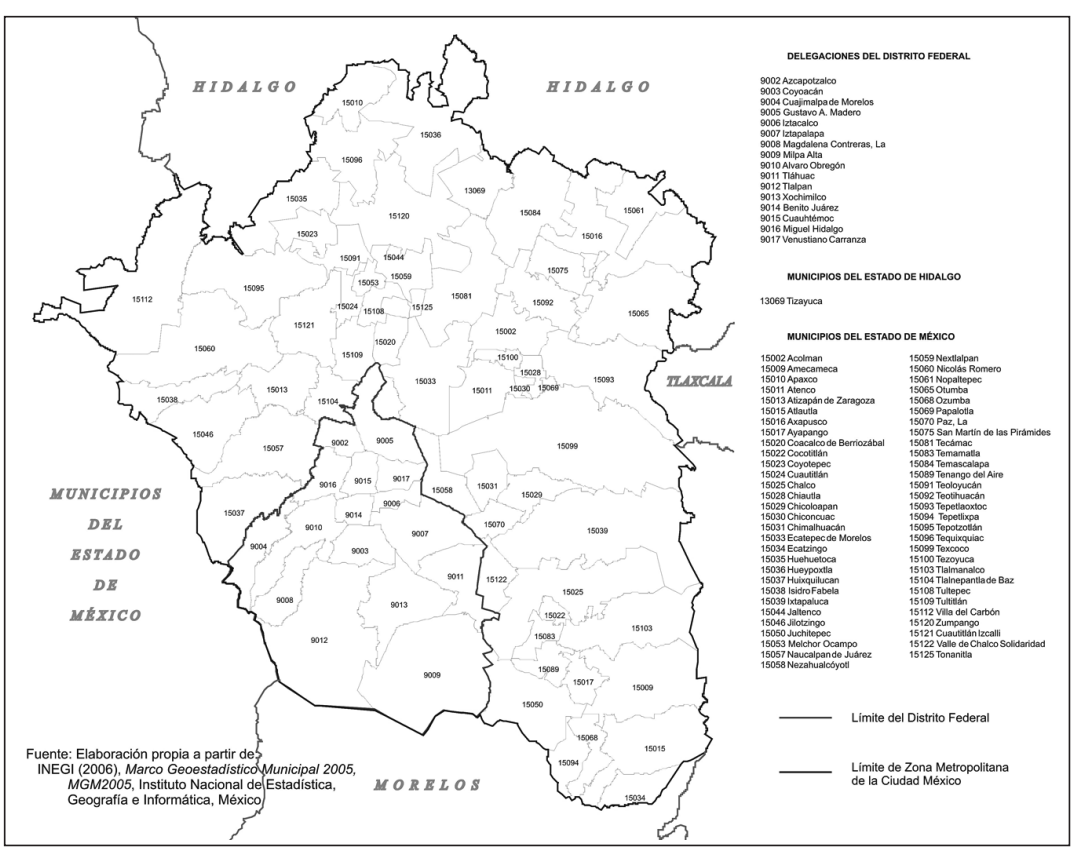

Para entender una relación funcional en la que $W$ está involucrada, es posible pensar en una variable explicativa (de un modelo) que está espacialmente determinada por "ella misma" (o espacialmente rezagada), es decir y de forma matricial: $W \boldsymbol{x}$, donde $W$ es una matriz de pesos espaciales $(\mathrm{NxN})$ y $\boldsymbol{x}$ es un vector $(\mathrm{Nx} 1)$, (Anselin, 2002). Esta variable consiste de una suma ponderada de valores de la variable $(\mathrm{x})$ en otros destinos $(j \neq i)$; para cada observación i,

$W x_{i}=\sum_{j \neq i} W_{i j} x_{j}$

Así, la cercanía-relevancia entre el lugar (i) y lugar (j) está representada en los pesos asignados $\left(\mathrm{w}_{\mathrm{ij}}\right)$. El tipo de pesos usados son determinados a priori, pero éstos pueden reflejar una distancia euclidiana entre dos lugares o una distance decay function (a mayor distancia, mayor el "costo") como el que comúnmente se usa en el modelo clásico gravitacional.

El uso de la ecuación (1) es útil para evaluar la interacción espacial entre áreas geográficas (cercanas) y la dependencia espacial que se genera sobre un proceso económico (en 
Marcos Valdivia L., Javier Delgadillo M. y Carlos Galindo P.

este caso, el empleo). Un primer uso estadístico de la ecuación (1) se puede dar a través de técnicas de exploración espacial con la finalidad de detectar autocorrelación espacial de una variable (en el presente estudio, empleo) entre unidades regionales. Al respecto, es posible detectar aglomeraciones a partir del uso de indicadores de autocorrelación espacial local como los delineados por Anselin (1995). Con esta técnica, las aglomeraciones (de empleo) son obtenidas cuando las unidades regionales despliegan autocorrelación espacial local que es estadísticamente significativa (es decir, no es aleatoria).

La presencia de estas aglomeraciones puede ser indicativa de la existencia de derramas (spillovers) geográficas, por ejemplo, las firmas en regiones vecinas que experimentan un rápido crecimiento del empleo estimulan un crecimiento del empleo en la región como respuesta. Asimismo, la ecuación (1) es utilizada en modelos de la econometría espacial (Fingleton, 2007) para estudiar directamente los efectos de derrama geográfica que producen las unidades regionales sobre procesos económicos específicos (empleo, difusión de tecnologías, crecimiento local, etcétera).

El objetivo inicial de la investigación consiste en reconocer qué factores pudieran estar impulsando derramas de empleo en la ZMCM y cómo han venido, incluso, alterando la creación y/o disminución de empleo durante los últimos años. Diversos son estos factores, pero una variable a la que se le podría poner especial interés es la inversión extranjera directa (IED). La IED puede ser vista como un medio para la adquisición de tecnología, esencial para impulsar la innovación en el desarrollo económico de una región; al respecto, la IED puede ser un canal de difusión de conocimiento y tecnología que contribuye a acelerar los procesos de desarrollo económico local a través de los efectos de derrama tecnológica o de conocimiento que tal variable podría generar. Pero también existen otros factores que quizás tienen un potencial mayor para generar derramas de conocimiento en las economías locales, por ejemplo, la inmigración de gente calificada o talentosa a una región (véase apartado 5 de este trabajo) o el establecimiento de universidades y centros de investigación.

Partiendo de esta consideración, la difusión territorial del conocimiento o de tecnologías (asociada a la IED, la inmigración de gente calificada, las universidades, etcétera) podrían ser elementos importantes detrás de la creación de una unidad de empleo en una micro-región de la ZMCM. En los apartados siguientes abordaremos empíricamente esta hipótesis a través de medir posibles derramas de empleo en la ZMCM por medio de técnicas estadísticas espaciales.

\section{ANÁLISIS DEL EMPLEO MANUFACTURERO Y DE SERVICIOS PROFESIONALES EN LA ZMCM}

Durante los últimos quince ańos la ZMCM ha perdido empleo manufacturero. Datos provenientes de los censos económicos indican que 942,289 trabajadores estaban empleados en el sector manufacturero en el año 1993, cifra que descendió a 885,764 para el año 2003; es decir, el empleo manufacturero decreció cerca de 6\% en la ZMCM en un periodo 
de 10 años. Para ubicar espacialmente este decremento del empleo manufacturero, se emplean técnicas de autocorrelación local (Anselin, 1995). En primer lugar se identifican aquellas aglomeraciones donde la diferencia en el nivel de empleo (difemp) manufacturero entre 1993 y 2003, presenta una autocorrelación espacial significativa con el difemp manufacturero de las delegaciones o municipios vecinos. ${ }^{2}$ También se muestra dónde está localizada la zona que ha registrado pérdidas en el empleo manufacturero y que además despliega autocorrelación espacial entre sus unidades sub-regionales. Como puede observarse en el Mapa 2, la pérdida del empleo manufacturero forma claramente una aglomeración espacial (véase conjunto de municipios con la etiqueta "aglomeración por abajo del promedio") conformada por los municipios de Tlalnepantla (15104) y Naucalpan de Juárez (15057) y las delegaciones Venustiano Carranza (9017), Miguel Hidalgo (9016), Cuauhtémoc (9015), Gustavo Madero (9005) y Azcapotzalco (9002).

Para contrastar lo que ha sucedido con el empleo en el sector manufacturero, en el Mapa 3 se muestran las aglomeraciones de la difemp pero ahora considerando las ramas de prestación de servicios profesionales-técnicos-especializados entre 1993 y 2003.

Mapa 2. ZMCM: Aglomeraciones espaciales del crecimiento del empleo manufacturero, 1993 y 2003.

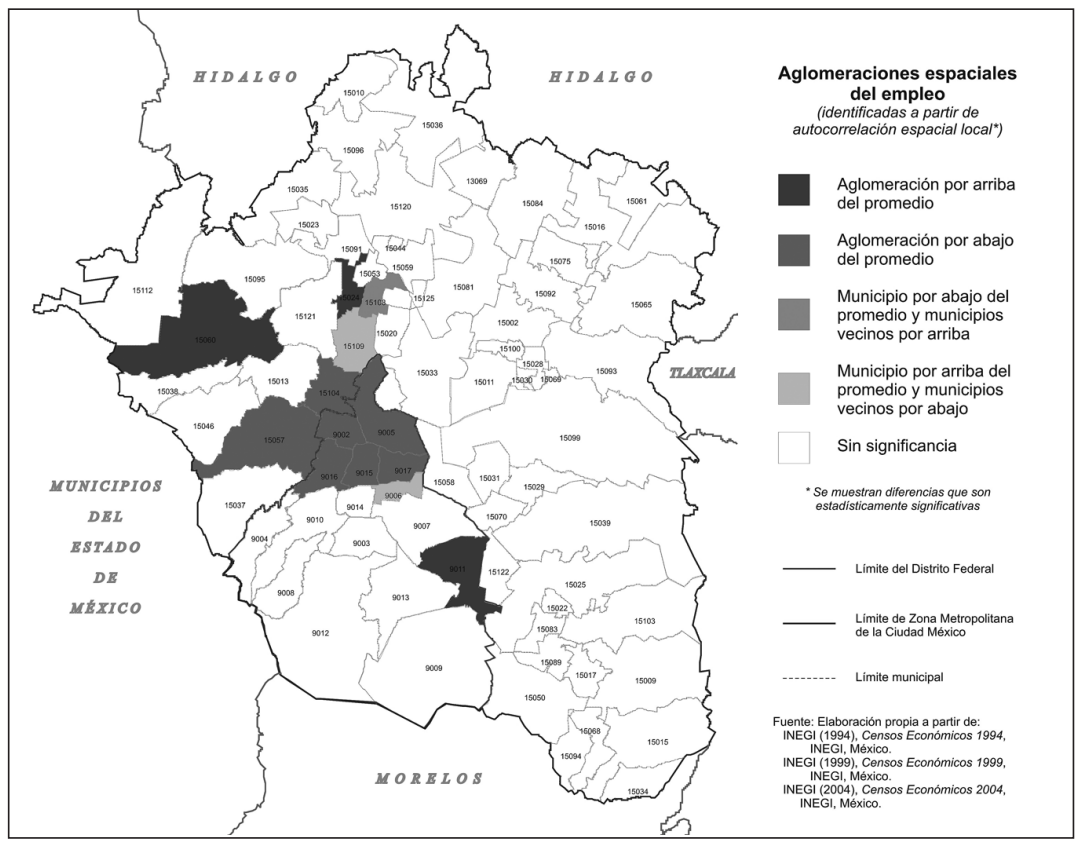

2 Las aglomeraciones que son derivadas en este estudio indican, en todos los casos, autocorrelación espacial local al 95\%. Se utilizaron métodos de tipo Monte Carlo para conducir los criterios de inferencia estadística utilizados en este documento. 
Marcos Valdivia L., Javier Delgadillo M. y Carlos Galindo P.

El mismo Mapa 3 muestra cómo la aglomeración dinámica en el crecimiento en el nivel de empleo en las ramas de servicio profesional (véase el conjunto de municipios con la etiqueta "aglomeración por arriba del promedio") tiene una clara ubicación en la zona poniente del Distrito Federal, donde destacan las delegaciones Cuajimalpa (9004), Álvaro Obregón (9010), Miguel Hidalgo (9016), Benito Juárez (9014), Coyoacán (9003), Cuauhtémoc (9015) e Iztacalco (9006), las cuales conforman el cluster más dinámico en el crecimiento del empleo de los servicios profesionales técnicos-especializados. ${ }^{3}$ También sobresale que al cluster dinámico anteriormente referido lo rodea un grupo de delegaciones, donde la difemp de las ramas de prestación de servicios profesionales se encuentra significativamente por debajo del promedio de la ZMCM. Destacan las delegaciones Gustavo A. Madero (9005) y Azcapotzalco (9002) donde la caída en el empleo manufacturero no ha sido compensada por un aumento (significativo espacialmente) del empleo en servicios profesionales, como fue el caso de las delegaciones Cuauhtémoc y Miguel Hidalgo (contrastar los Mapas 2 y 3 ).

Mapa 3. ZMCM: Aglomeraciones espaciales del crecimiento del empleo en el sector servicios, 1993 y 2003.

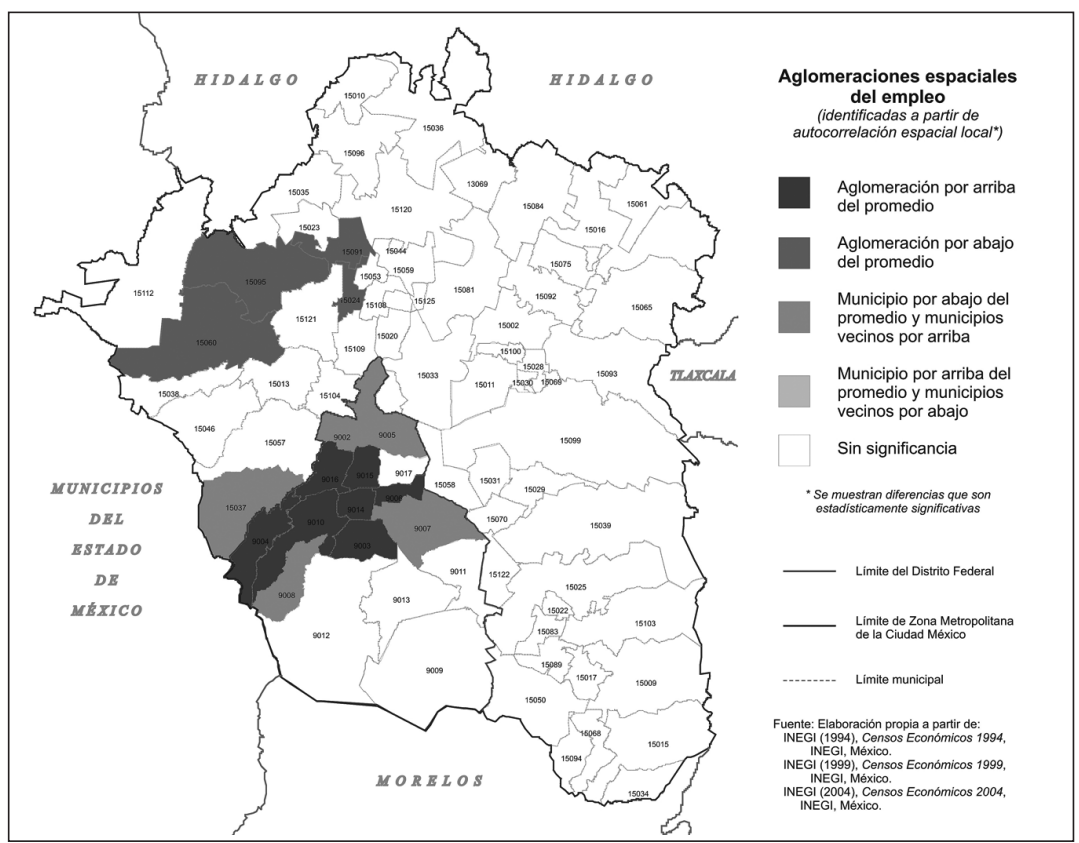

3 Es interesante destacar que la IED se concentra en las delegaciones Miguel Hidalgo, Cuauhtémoc, Benito Juárez y Álvaro Obregón que aglomeran la mayor parte de las empresas con IED (Correa, 2003: 113, 118). 


\section{LA INFLUENCIA DE LOS SECTORES CREATIVOS Y TÉCNICOS EN LA FORMACIÓN DE LOS NUEVOS CLUSTERS DE EMPLEO}

Algunos autores han llamado la atención sobre el análisis de los cambios territoriales observados durante los últimos ańos en el sector de servicios en la ZMCM (Garza, 2006). Éste es un punto importante por estudiar ya que autores como Hanson (1998) han sostenido que la ZMCM se ha des-industrializado a consecuencia de la apertura comercial, lo que ha originado un desplazamiento de la manufactura hacia el norte del país. La pérdida del sector manufacturero puede documentarse a partir de ejercicios con las cifras de empleo y producción de la región (Sobrino, 2007). Como consecuencia, este proceso de pérdida del sector manufacturero en la ZMCM ha venido acompañado por un incremento en el empleo y la producción en las ramas asociadas al sector servicios.

Los efectos espaciales que pueden estar asociados al nuevo patrón de "terciarización" de la ZMCM (véase Mapa 3) muy bien podrían responder a tipos específicos de actividades en el sector servicios que podrían estar generando derramas. Para desarrollar esta idea, se realizó un ejercicio de segmentación del empleo en el sector servicios (considerando sólo la rama 541 de servicios profesionales y técnicos según la clasificación de ocupaciones del Censo Económico 2004), en el cual se identificaron a las subramas que pueden catalogarse como clase creativa y clase técnica.

La motivación teórica de esta segmentación proviene del trabajo de Florida (2005) quien introduce la noción de clase creativa o (capital creativo) para analizar las relaciones entre las ciudades y el crecimiento económico. Desde la óptica de esta investigación, la clase creativa tiene el potencial -incluso mayor que otras variables tradicionales como lo es la IED- de generar derramas en el empleo.

Esta visión de la clase creativa difiere de la teoría del capital humano de Lucas (1988) por dos razones: a) se identifica un tipo de capital humano, gente creativa (no necesariamente relacionada con niveles de educación), que es central para el crecimiento económico, y b) se atiende especialmente a los factores que determinan las decisiones de ubicación de esa clase creativa (Ibidem, p. 34). Destacamos este punto b) porque esta teoría no considera la localización de las firmas, sino la localización de la gente para radicar o migrar (situación que mezcla oportunidades económicas y estilos de vida). En este sentido, la noción de clase creativa ofrece otra explicación al por qué de la presencia de aglomeraciones, es decir, las firmas al agruparse sacan provecho de las concentraciones de gente talentosa.

Para construir la variable de clase creativa y la clase técnica se consideraron una serie de subramas descritas en el Cuadro $1 .{ }^{4}$ Como acotación, debe observarse que estas subramas no son necesariamente las que emplean trabajadores con elevada calificación en el área técnica y/o científica.

4 Al respecto, se consideraron algunos de los lineamientos de clasificación propuestos por Fingleton (2007). 
Marcos Valdivia L., Javier Delgadillo M. y Carlos Galindo P.

Cuadro 1: Clasificación de la Clase Creativa y Técnica a partir de los Censos Económicos

\begin{tabular}{l}
$\qquad$ Clase creativa \\
\hline Rama 5414 Diseño especializado \\
Rama 5415 Servicios de consultoría en computación \\
Rama 5418 Servicios de publicidad y actividades relacionadas \\
Subrama 54132 Servicios de arquitectura de paisaje y urbanismo \\
Subrama 54134 Servicios de dibujo \\
Subrama 54172 Servicios de investigación y desarrollo en ciencias sociales y humanidades \\
Subrama 54191 Servicios de investigación de mercados y encuestas de opinión pública \\
Subrama 54192 Servicios de fotografía \\
Subrama 54193 Servicios de traducción e interpretación \\
Clase téennica
\end{tabular}

Subrama 54133 Servicios de ingeniería

Subrama 54136 Servicios de levantamiento geofísico

Subrama 54138 Laboratorios de pruebas

Subrama 54162 Servicios de consultoría en medioambiente

Subrama 54169 Otros servicios de consultoría cientifica y téenica

Subrama 54171 Servicios de investigación y desarrollo en ciencias físicas, de la vida e ingeniería

El Cuadro 2 muestra algunos resultados regionales del comportamiento del sector 541 para el año 2003. Primero, destaca el hecho de que la productividad agregada del sector (138.6) se encuentra muy por debajo de la productividad agregada que

Cuadro 2: Sector 541. Servicios profesionales y técnicos

\begin{tabular}{|c|c|c|c|c|c|c|c|}
\hline & Unidades económicas & $\%$ & Valor agregado & $\%$ & Empleo & $\%$ & $\operatorname{Pr}$ \\
\hline País & 68,589 & 100 & $65,478,697$ & 100 & 472,348 & 100 & 138.6 \\
\hline DF & 11,019 & 0.16 & $30,361,935$ & 0.46 & 179,503 & 0.38 & 169.1 \\
\hline ZMCM & 15,318 & 0.22 & $32,793,801$ & 0.5 & 206,121 & 0.44 & 159.1 \\
\hline Todo el país y los sectores & $3,005,157$ & & $3,217,290,004$ & & $16,239,536$ & & 198.1 \\
\hline Sector 541 & & & & 0.02 & & 0.03 & \\
\hline ZMCM & & & & 0.01 & & 0.01 & \\
\hline \multicolumn{8}{|c|}{ Clase creativa y técnica (datos respecto a la ZMCM) } \\
\hline & & & & & Empleo & $\%$ & $\operatorname{Pr}$ \\
\hline & & & Clase creativa ZMCM & & 75,310 & 0.37 & 134.1 \\
\hline & & & Clase creativa DF & & 64,629 & 0.31 & 146 \\
\hline & & & Clase técnica ZMCM & & 20,772 & 0.1 & 206.9 \\
\hline & & & Clase técnica DF & & 18,882 & 0.09 & 211.8 \\
\hline
\end{tabular}


incluye a todos los sectores (198.1). El sector 541 contribuye con $2 \%$ del total de empleo del país, siendo la ZMCM la que concentra el mayor porcentaje del empleo en este sector (1\%).

No obstante lo anterior, el sector 541 es heterogéneo por sí mismo y al descomponer este sector en una clase creativa y una clase técnica se destacan los siguientes aspectos: a) la productividad del sector técnico es alta (incluso por arriba de la media nacional); mientras que, b) el sector creativo mostró tener una menor productividad, quizás similar a la observada en el agregado nacional. Sin embargo, los efectos a nivel local -de ambos sectores- podrían diferir del comportamiento observado en el agregado de la ZMCM. Para analizarlo, replicamos nuevamente el ejercicio de autocorrelación espacial local para identificar los "clusters" de empleo de la clase creativa y la clase técnica (véanse Mapas 4 y 5). El resultado central es que ambos clusters dinámicos son muy similares (véase el conjunto de municipios con la etiqueta "aglomeración por arriba del promedio"), es decir, si existieran efectos de derrama de empleo, éstos estarían operando tanto en la clase técnica (que está más asociada al capital humano) como también en la denominada "clase creativa."

Mapa 4. Clusters, clase técnica (2004)

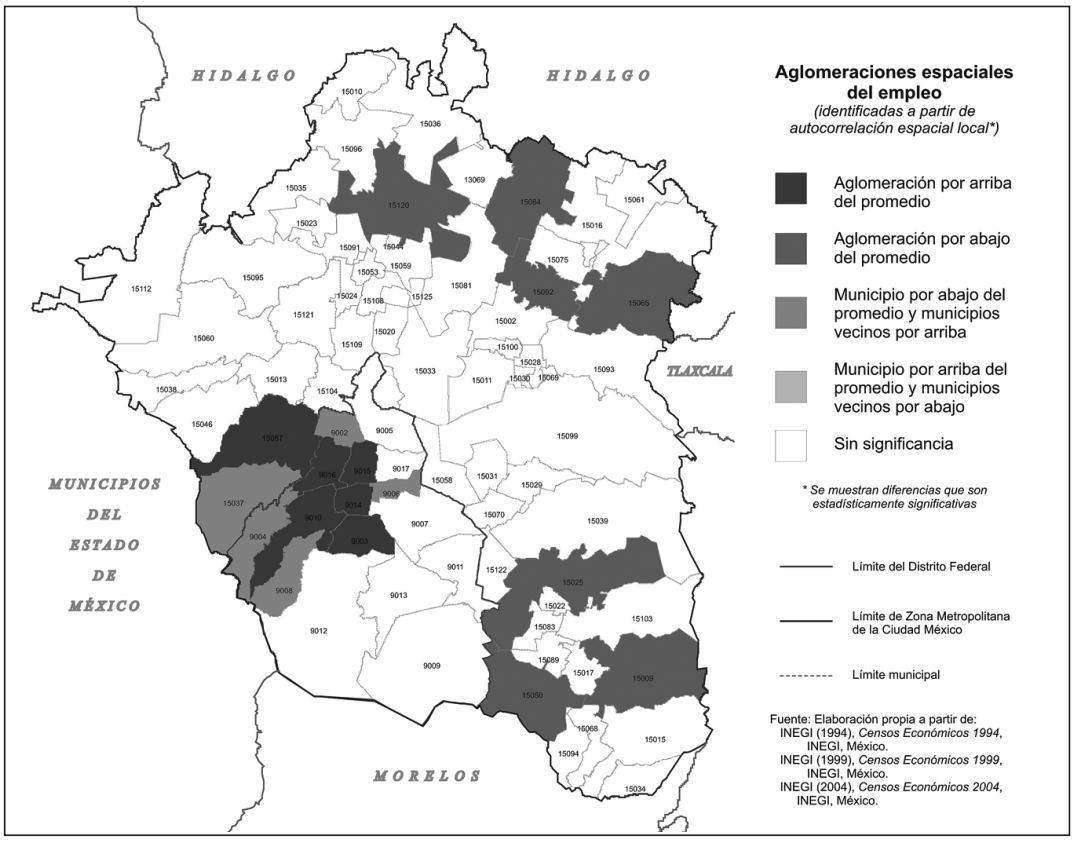


Marcos Valdivia L., Javier Delgadillo M. y Carlos Galindo P.

Mapa 5. Clusters, clase creativa (2004)

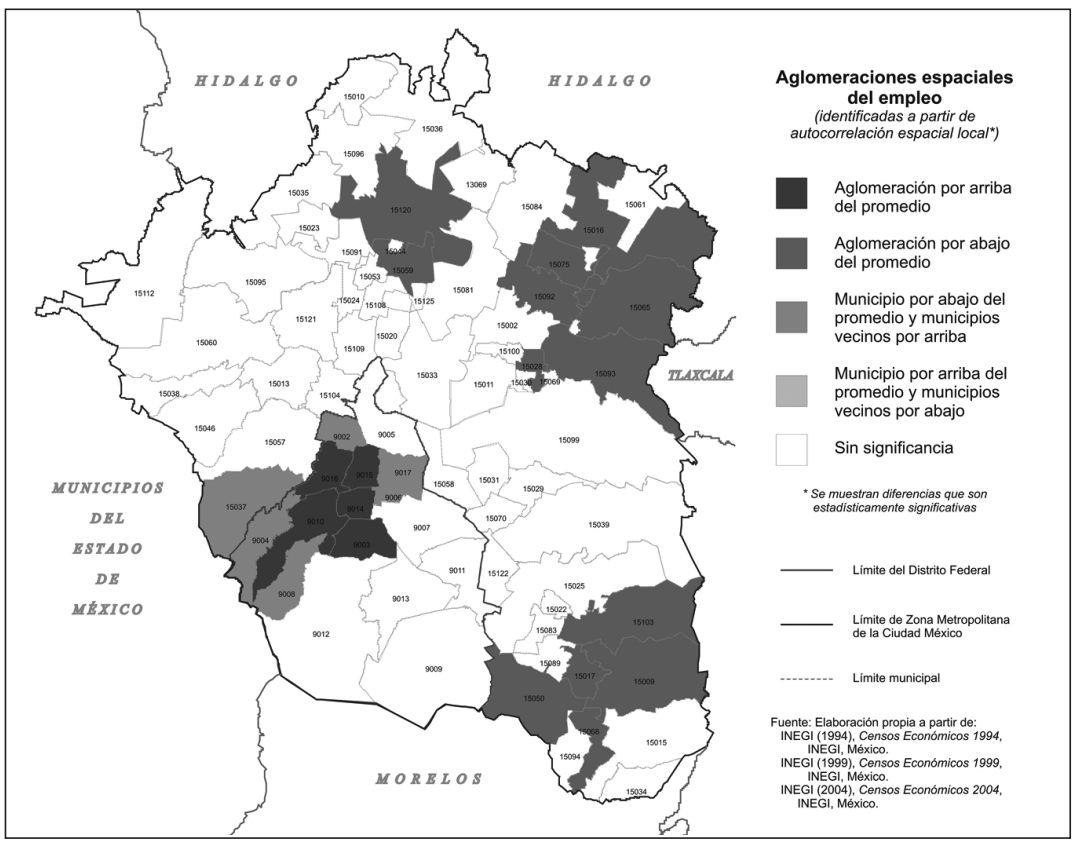

\section{MODELO ECONOMÉTRICO QUE EXPLICA \\ EL CRECIMIENTO DEL EMPLEO DE SERVICIOS \\ PROFESIONALES-TÉCNICOS-ESPECIALIZADOS \\ A PARTIR DE DERRAMAS}

En el Mapa 3 se ilustró la presencia de aglomeraciones en la creación de empleo en el sector de servicios profesionales-técnicos especializados (1993-2003). Y, en particular, la aglomeración destacable fue la localizada en la parte poniente del Distrito Federal. En los Mapas 4 y 5 mostramos que esta aglomeración está también presente, tanto en el nivel de empleo (2003) de la clase técnica como en la clase creativa, es decir, ambos procesos pudieran estar explicando la presencia de derramas en el sector profesional de servicios.

En este apartado se analizará el crecimiento en el nivel de empleo (difemp) en el sector de servicios profesionales y técnicos (como agregado) en el periodo1999-2003 y se procederá a aplicar una serie de modelos econométricos para evaluar si efectivamente hay presencia o no de derramas como las sugeridas en los Mapas 3, 4 y 5. 
Primero partimos del cálculo de un índice de Moran $^{5}$ de la difemp con el propósito de evaluar si hay presencia de dependencia espacial global en la variable en el nivel municipal/delegacional. El índice de Moran obtenido fue de 0.3335 , valor que es estadísticamente significativo, lo que nos permite señalar que es improbable que el patrón territorial que despliega la variable sea aleatorio. Esta dependencia espacial en la difemp puede ser indicativa de que muchos factores comparten una característica espacial, pero no necesariamente implica efectos de interacción espacial (entre áreas geográficas) o de derramas como los buscados en este trabajo. Para analizar más en detalle este punto, se realizaron los siguientes ejercicios econométricos.

En primer término analizamos los efectos de aglomeración (de las firmas) sobre la creación de empleo a través del siguiente indicador de intensidad del cluster (aglomeración horizontal):

$C I=\frac{E_{i}}{A}$

donde $E$ es el empleo del sector en el área $i$. y $A$ representa la superficie del municipio o delegación $\left(e n \mathrm{Km}^{2}\right) i$. Este índice captura las externalidades positivas (derramas) asociadas a las economías de aglomeración.

Para evaluar si las externalidades están asociadas al crecimiento de empleo, se propuso la siguiente ecuación:

difemp $_{\text {Serv }}=a C I+b C I^{2}+c E d u c a c i o ́ n+\varepsilon$

$C T^{2}$ rescata los posibles efectos de "congestión" que puede generar una concentración elevada de firmas; Educación es una variable proxy de capital humano y que en este trabajo se concibe como los años promedio de escolaridad de la población; $a, b$ y $c$ son parámetros por estimar. Las hipótesis son las siguientes: 1) si $a$ es positivo, existen externalidades positivas en el empleo, y 2) si $b$ es negativo, existen efectos de congestión.

Los resultados de la estimación por mínimos cuadrados ordinarios se muestran en el cuadro 3.

La interpretación de los resultados lleva a inferir la presencia de externalidades positivas presentes en la creación de empleo, pero sin que existan efectos de congestión ( $b$ no es diferente de cero). La variable de educación sólo es significativa al 10\%, lo que sugiere un papel menor de la variable en el crecimiento del empleo. De esta manera, la dependencia espacial (véase índice de Moran) que se encuentra en el difemp a nivel municipal, puede ser atribuida básicamente a la aglomeración de unidades económicas del sector en el área geográfica, las cuales pueden desarrollar derramas, ya que no prevalece autocorrelación espacial en el término de error de la ecuación (3). Por lo tanto, no se

5 Se utilizó una matriz W de primer orden para el cálculo del índice. 
Marcos Valdivia L., Javier Delgadillo M. y Carlos Galindo P.

\begin{tabular}{|c|c|c|c|}
\hline \multicolumn{2}{|c|}{ Variable } & \multicolumn{2}{|c|}{ Coeficiente } \\
\hline \multirow{2}{*}{\multicolumn{2}{|c|}{ Constante }} & \multirow{2}{*}{\multicolumn{2}{|c|}{$\begin{array}{c}-2637.36 \\
(1897.32)\end{array}$}} \\
\hline & & & \\
\hline \multirow{2}{*}{\multicolumn{2}{|c|}{ Clservicios1999 }} & \multirow{2}{*}{\multicolumn{2}{|c|}{$\begin{array}{c}8.99 \\
(3.27)^{\star \star \star}\end{array}$}} \\
\hline & & & \\
\hline \multirow{2}{*}{\multicolumn{2}{|c|}{ Clservicios²1999 }} & & \\
\hline & & \multicolumn{2}{|c|}{$\begin{array}{c}0.00 \\
(0.001)\end{array}$} \\
\hline \multicolumn{2}{|c|}{ cEducación2000 } & \multicolumn{2}{|c|}{$\begin{array}{c}409.17 \\
(241.70)^{\star \star}\end{array}$} \\
\hline Criterios & GL & Valor & \\
\hline$R^{2}$ & 77 & 0.5401 & \\
\hline $\mathrm{R}^{2}$ Ajustado & 77 & 0.5222 & \\
\hline Log Likelihood & 77 & .754 .1230 & \\
\hline \multicolumn{4}{|c|}{ Pruebas de dependencia espacial } \\
\hline Prueba & $\overline{G L}$ & Valor & Probabilidad \\
\hline I de Moran & -0.0618 & -0.5729 & 0.5667 \\
\hline Multiplicador Lagrange (lag) & 1 & 0.6330 & 0.4263 \\
\hline LM Robusto & 1 & 6.4834 & 0.0109 \\
\hline Multiplicador Lagrange (error) & 1 & 0.6995 & 0.4029 \\
\hline LM Robusto (error) & 1 & 6.5500 & 0.0105 \\
\hline Multiplicador Lagrange (SARMA) & 2 & 7.1830 & 0.0276 \\
\hline
\end{tabular}

$\star \star \star$ Significativo al $99 \%$

$\star *$ Significativo al $90 \%$

encontraron derramas entre áreas geográficas pero sí al interior de las mismas; sin embargo, es importante señalar que esta ausencia de derramas entre unidades sub-regionales puede ser debida al nivel de agregación del estudio.

Los patrones espaciales detectados en el empleo de servicios tienden a reproducirse con el empleo manufacturero pero con importantes diferencias. Se analizó la difemp manufacturera entre los ańos 1993 y 2003 , y se aplicaron el mismo tipo de estimaciones que para el caso de la difemp de servicios profesionales. La difemp manufacturera también despliega dependencia espacial significativa pero a diferencia de lo que pasa con la difemp de servicios, la dependencia espacial se refleja en agrupamientos de delegaciones con pérdida de empleo (véase Mapa 2). Asimismo, se estimó la ecuación (3) para la difemp manufacturera, los resultados se muestran en el cuadro 4 .

Como lo indica el coeficiente negativo asociado al "cluster horizontal”, la estimación sugiere la presencia de externalidades negativas en la generación de empleo manufacturero en la ZMCM. En general, este resultado era de esperarse; sin embargo, al observar 
Nuevos patrones espaciales en las derramas de empleo

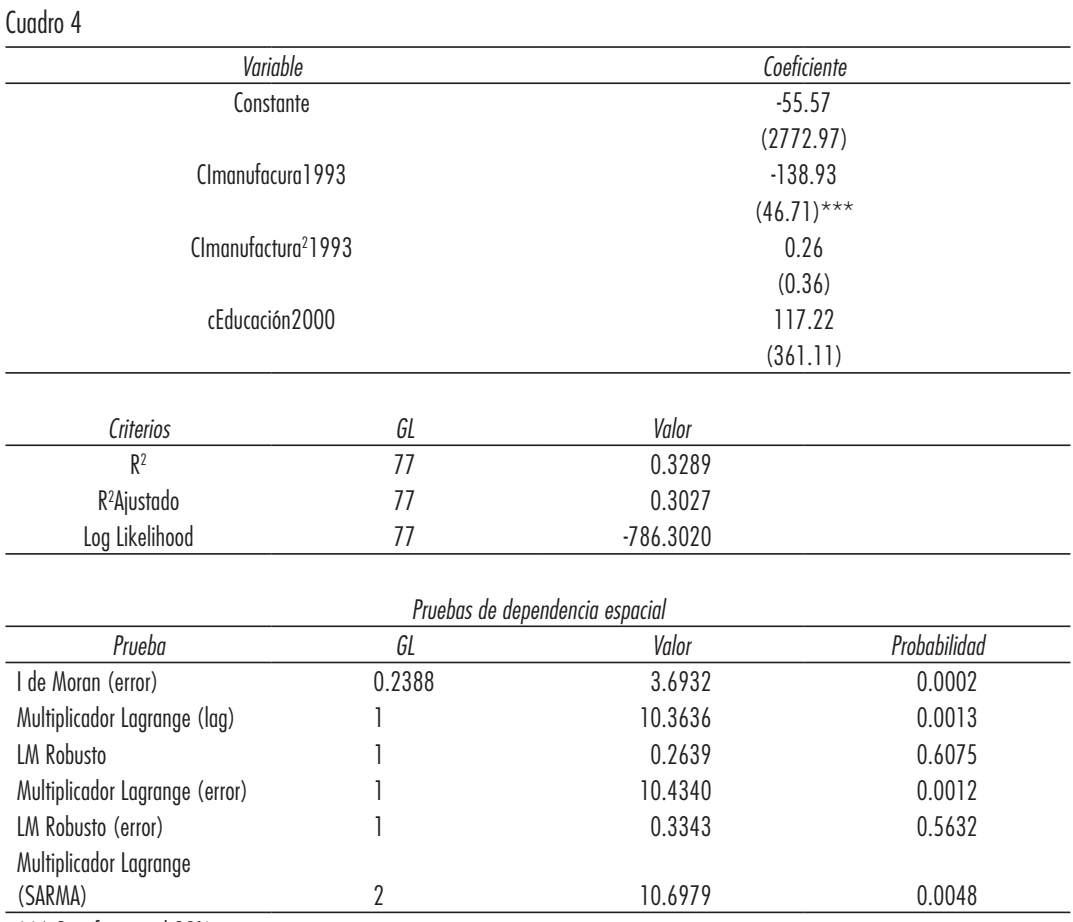

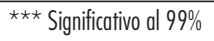

el cuadro 4 destaca que los errores de la regresión continúan mostrando fuerte dependencia espacial. Este resultado difiere de lo encontrado cuando se considera a la difemp de servicios, ya que en ese caso, considerar el "cluster horizontal" $(\mathrm{CH})$ es suficiente para absorber todos los posibles efectos de derrama que se generan a ese nivel de agregación territorial. Pero, para el caso del empleo manufacturero, esto no ocurre porque prevalecen efectos de dependencia espacial que no están asociados a la concentración de unidades económicas. Por lo que indican las pruebas de diagnóstico de autocorrelación de los errores de la regresión, es muy probable que el modelo esté insuficientemente especificado y requiera de otras variables que no están incluidas en el modelo (y que den cuenta de esa dependencia espacial).

Asimismo, las pruebas también sugieren un modelo autorregresivo para corregir la dependencia espacial. Éste es un caso interesante para los propósitos centrales de esta investigación, porque estaría poniendo a prueba la hipótesis de que también pudieran existir derramas entre las unidades sub-regionales. Con el propósito de ilustrar este 
Marcos Valdivia L., Javier Delgadillo M. y Carlos Galindo P.

punto (ya que las pruebas estadísticas de dependencia espacial no son contundentes al respecto), se estimó el siguiente modelo autorregresivo:

difermp $_{\text {Manufactura }}=\rho W$ difemp $_{\text {Manufactura }}+a C I+b C I^{2}+c E$ ducación $+\varepsilon$

Los resultados se muestran en el cuadro 5 .

En este caso, los resultados del modelo autorregresivo sugieren claramente que existen derramas entre los municipios (delegaciones) y que incluso son más importantes que las presentadas al interior de los mismos (observe, asimismo, que $\mathrm{CH}$ no alcanza a ser significativa al 95\%). Estos resultados podrían sugerir que los efectos de derrama en el sector manufacturero no requieran de interacciones tan cercanas como las que podrían estar ocurriendo, en contraste, en el sector de servicios. Es por ello que la interacción espacial entre municipios (delegaciones) se muestra, en los modelos analizados, significativa para el empleo del sector manufacturero y no así para el empleo del sector servicios.

Cuadro 5

\begin{tabular}{|c|c|c|c|c|}
\hline \multicolumn{2}{|c|}{ Variable } & \multicolumn{3}{|c|}{ Coeficiente } \\
\hline \multirow{2}{*}{\multicolumn{2}{|c|}{ Constante }} & & -844.24 & \\
\hline & & & (2422.21) & \\
\hline \multicolumn{2}{|c|}{ DifempManufactura 1993} & & 0.54 & \\
\hline & & & $(0.11)^{\star \star \star}$ & \\
\hline \multicolumn{2}{|c|}{ CImanufacural993 } & & -81.90 & \\
\hline & & & $(42.78)^{\star \star}$ & \\
\hline \multicolumn{2}{|c|}{ CImanufactura ${ }^{2} 1993$} & & 0.09 & \\
\hline & & & $(0.32)$ & \\
\hline \multicolumn{2}{|c|}{ cEducación2000 } & & 184.61 & \\
\hline & & & $(315.06)$ & \\
\hline Criterios & GL & Valor & & \\
\hline $\mathrm{R}^{2}$ & 76 & 0.4635 & & \\
\hline $\mathrm{R}^{2}$ Ajustado & 76 & nd & & \\
\hline Log Likelihood & 76 & -780.0550 & & \\
\hline \multicolumn{5}{|c|}{ Pruebas de dependencia espacial } \\
\hline Prueba & GL & Valor & & Probabilidad \\
\hline Likelihood Ratio & 1 & 12.4943 & & 0.0004 \\
\hline
\end{tabular}


Este último punto permite proponer la siguiente hipótesis: el proceso de terciarización en la ZMCM ha condicionado a las derramas (de empleo) a operar en distancias cortas de interacción espacial. La comprobación de esta hipótesis requiere de una mayor investigación basada en unidades regionales más desagregadas, por ejemplo, Áreas Geoestadísticas Básicas (AGEBs).

\section{CONCLUSIONES}

En este trabajo se analizaron los efectos derrama sobre el empleo asociados a las aglomeraciones económicas al interior de la ZMCM. El objetivo fue evaluar si tanto el crecimiento del empleo formal, en el sector manufacturero como en el sector de servicios profesionales, está bajo influencia de externalidades espaciales. Las técnicas utilizadas permiten concluir que hay evidencia de derramas en la creación o destrucción de empleo durante los últimos ańos. En este sentido se detecta que el empleo en el sector manufacturero ha sido sujeto a externalidades negativas, mientras que las externalidades positivas han operado para el caso del empleo del sector de servicios profesionales.

Asimismo, la presencia de los efectos derrama sobre el empleo en los sectores estudiados está en función de la localización geográfica de las aglomeraciones de empleo respectivas. De esta manera, los resultados revelan que hay una descongestión del sector manufacturero en el norte del Distrito Federal, mientras se observa una nueva aglomeración del sector servicios en la parte poniente, en particular, en las delegaciones Cuajimalpa, Álvaro Obregón y Miguel Hidalgo.

Finalmente, un diagnóstico espacial de los resultados obtenidos permite concluir que el proceso acelerado de terciarización que ha experimentado la ZMCM durante las últimas dos décadas, ha condicionado que los efectos derrama de empleo operen en distancias cortas de interacción espacial. Esta hipótesis podría tener fuertes implicaciones en el entendimiento de la dinámica de polarización económica que ha sufrido la ZMCM en años recientes, pues al depender ahora las derramas de empleo en distancias más cortas, mayor es la probabilidad de que generen dinámicas territoriales de desigualdad en la creación de nuevos empleos. A nuestro entender, este resultado debería ser internalizado en el diseño de las políticas públicas orientadas a promover el empleo urbano, ya que el alcance y efectividad de tales políticas pueden verse afectadas ante el cambio de patrón en las derramas de empleo que ha sufrido la Ciudad de México en los últimos años.

Sin embargo, somos concientes que para comprobar esta hipótesis de cambio en el patrón de derramas en la ZMCM, es necesario profundizar las evidencias realizando una investigación más detallada con unidades micro-regionales como podrían ser las AGEBs. 
Marcos Valdivia L., Javier Delgadillo M. y Carlos Galindo P.

\section{BIBLIOGRAFÍA}

Anselin, L., "Local Indicators of Spatial Association-LISA", Geographical Analysis, 27, 1995, pp. 93-113.

Anselin, L., "Under the Hood. Issues in the Specification and Interpretation of Spatial Regression Models", Agricultural Economics, 27, 2002, pp. 247-267.

Anselin, L., "Spatial Externalities", International Regional Science Review, 26, 2003, pp. $147-152$

Asuad, N. y Luis Quintana, “Convergencia espacial y concentración regional agrícola en México 1970-2003”, Problemas del Desarrollo, núm. 149, abril 2007.

CEPAL, La IED y las capacidades de innovación y desarrollo locales: lecciones del estudio de los casos de la maquila automotriz y electrónica en Ciudad Juárez. Documento LC/ MEX/L.604, Santiago de Chile, 2004.

CONAPO, Escenarios demográficos y urbanos de la Zona Metropolitana de la Ciudad de México, 1990-2010, Consejo Nacional de Población, México, 1998.

CONAPO, Delimitación de las zonas metropolitanas de México 2005, Consejo Nacional de Población, México, 2007.

Correa, Ma. A., "Inversión extranjera directa en la región Centro de México, 19942000”, en: Villareal, D., D. Mignot y D. Hiernaux (coords.), Dinámicas metropolitanas y estructuración territorial. Estudio comparativo México-Francia, UAM-X y Miguel Ángel Porrúa, México, 2003, pp. 107-125.

Covarrubias, F., "Crecimiento metropolitano de la Ciudad de México y necesidades de financiamiento", Programa Universitario de Estudios sobre la Ciudad, UNAM, 2000.

Díaz, A., "Un modelo de aglomeraciones, inversión extranjera y crecimiento para la nueva geografía económica de México." Observatorio de la Economía Latinoamericana, Universidad de Málaga, núm. 16, noviembre 2003.

Fingleton, B., "Externalities, Economic Geography, and Spatial Econometrics: Conceptual and Modeling Developments", International Regional Science Review 26, 2003, pp.197-207.

Fingleton, B. et al, "Employment Growth and Cluster Dynamics", en The Economic Geography of Innovation, editado por Karen R. Polenske, Cambridge: Cambridge University Press, 2007, pp. 60-84.

Florida, R., Cities and the Creative Class, NY: Routledge, 2005.

Fujita, M., Krugman, P., y A. Venables, The Spatial Economy, Massachusetts: The MIT Press, 1999.

Gaceta Oficial del Distrito Federal, "Declaratoria de la Zona Metropolitana del Valle de México", 23 de enero de 2005.

Garza, G, coordinador, La organización espacial del sector servicios en México, México, El Colegio de México, 2006.

Garza, G., Macroeconomía del sector servicios en la Ciudad de México, SEDECO, GDF, 2007. 
Hanson, G. H., "North American Economic Integration and Industry Location", Oxford Review of Economic Policy, 14(2), 1998, pp.30-44.

Lucas, R., "On the Mechanics of Economic Development", Journal of Monetary Economics, 22, 1988, pp. 3-42.

Marshall, A., Principles of Economics, London: Macmillan, 1920.

Pacheco, R., "Una crítica al paradigma de desarrollo regional mediante clusters industriales forzados", Estudios Sociológicos, vol. xxv, núm. 75, El Colegio de México, 2007, pp. 683-707.

Parnreiter, C., Revista EURE, vol. xxxI, núm. 92, Santiago de Chile, 2005, pp. 5-28.

Partida, V., Escenarios demográficos de la ciudad de México, unam, 2000.

Pérez, O. E., "La inversión extranjera directa y la transferencia de tecnología", 2006. http://nodo50.org/cubasigloXXI/economia/perezvi_280206.pdf

Porter, M. E., The Competitive Advantage of Nations, London: McMillan, 1990.

Pradilla, E., "Fragmentación y exclusión en la megalópolis mexicana". Revista Nueva Sociedad, núm. 156, julio-agosto, 1998, pp. 180-193.

Ramírez, B., "Del funcionalismo industrial al funcionalismo de los servicios: ¿La nueva metrópoli postindustrial del valle de México?", Revista EURE, vol. XXXII, núm. 95, Santiago de Chile, 2006, pp. 61-74.

Romo, D., "Derramas tecnológicas de la inversión extranjera en la industria mexicana". Revista Comercio Exterior, vol 53, núm. 3, México, 2003.

Ruiz, D., Dimensión territorial del desarrollo económico de México. Facultad de Economía de la UNAM, México, 2005.

Sobrino, J., "Desempeño industrial en las principales ciudades de México, 1980-2003", Estudios Demográficos y Urbanos, 22(2), 2007, pp. 243-290.

Vázquez, A., "Surgimiento y transformación de clusters y milieus en los procesos de desarrollo", Revista EURE, vol. xxxII, Núm. 95, Santiago de Chile, 2006, pp. 75-92. 
